\title{
КОРРЕКЦИЯ ИНТРААБДОМИНАЛЬНОЙ ГИПЕРТЕНЗИИ И ВЫБОР СПОСОБА ЗАВЕРШЕНИЯ ОПЕРАЦИИ ПРИ РАСПРОСТРАНЕННОМ ПЕРИТОНИТЕ
}

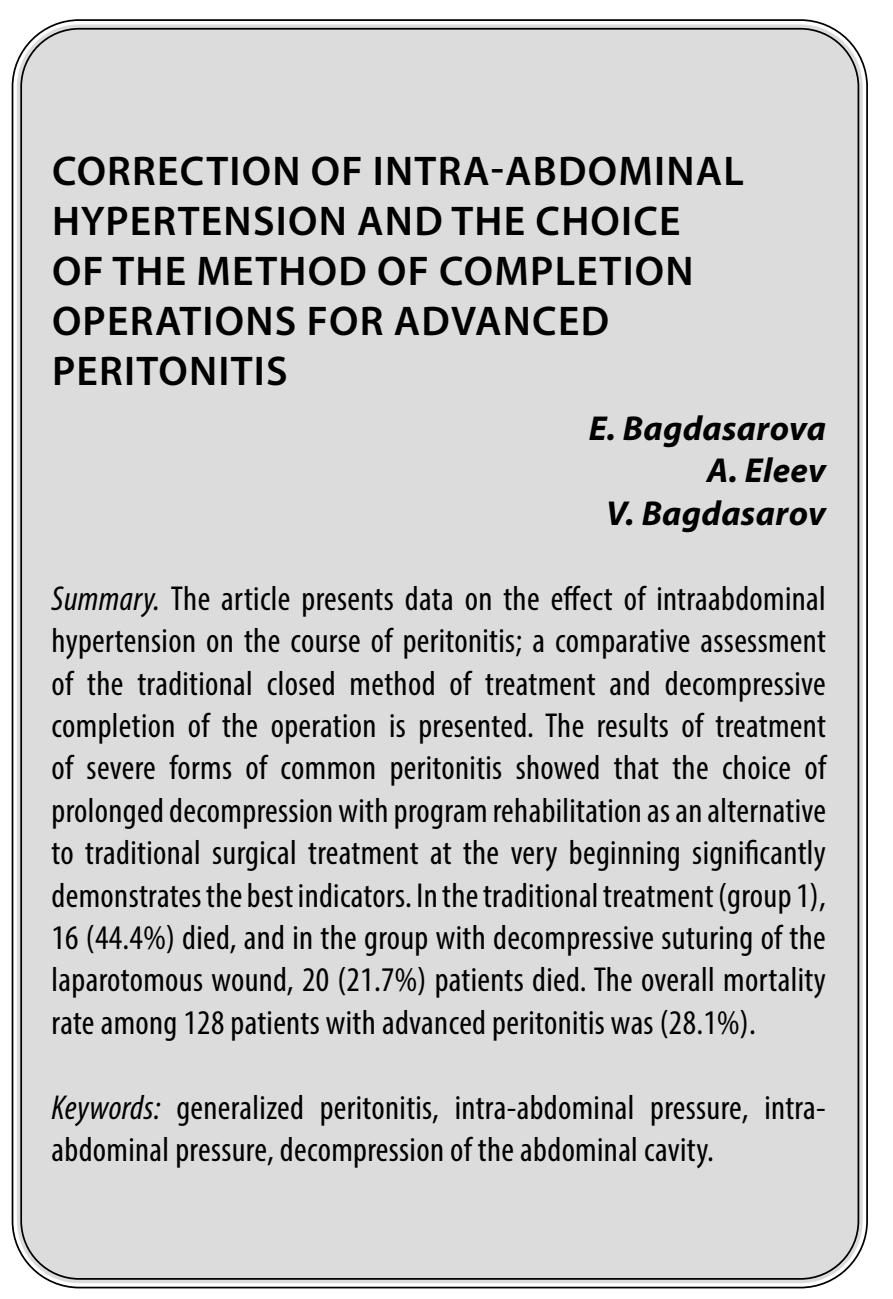

A нализ научной литературы, посвященной программным релапаротомиям и этапным санациям при перитоните свидетельствует о недооценке интраабдоминальной гипертензии (ИАГ) и о невостребованной коррекции ИАГ при распространенном перитоните (РП) [1, с. 74; 9, с. 147]. За последние годы появились сообщения, комментирующие показания к выбору этапного метода лечения, одним из которых является уровень внутрибрюшного давления [7, с. 69; 6].

У больных распространенным перитонитом, по данным этих авторов, ИАГ развивается у 30 и более процентов, а летальность от синдрома интраабдоминальной гипертензии (СИАГ) достигает очень высоких цифр- 42-68\%.
Багдасарова Елена Анатольевна

Д.м.н., заведующая отделением, ДЗМ ГКБ С.С. Юдина

Елеев Алим Анатольевич

Врач хирург, колопроктолог, онколог, ДЗМ ГКБ

С.C. Юдина

Alimeleev@gmail.com

Багдасаров Валерий Вартанович

Д.м.н., профессор, Российский Университет Дружбы

Народов

Аннотация. В статье приведены данные о влиянии интраабдоминальной гипертензии на течение перитонита; представлена сравнительная оценка традиционного закрытого способа лечения и декомпрессивного завершения операции. Результаты лечения тяжелых форм распространенного перитонита показали, что выбор в самом начале тактики пролонгированной декомпрессии с программными санациями как альтернатива традиционному хирургическому лечению достоверно демонстрируют лучшие показатели. При традиционном лечении (1-я группа) умерли 16 (44,4\%), в группе с декомпрессивным ушиванием лапаро-томной раны умерли 20 $(21,7 \%)$ больных. Общая летальность среди 128 больных с распространенным перитонитом составила $(28,1 \%)$.

Ключевые слова: распространенный перитонит, внутрибрюшное давление, интраабдоминальная гипертензия, декомпрессия брюшной полости.

Многочисленные публикации описывают влияние ИАГ на различные системы органов в большей или меньшей степени и на весь организм в целом [12; 13, с. $12 ; 14$, с. $389 ; 18$, с. $21 ; 19$, с. 512 ].

Уже доказано [24, с. 14], что прогрессирование ИАГ у больных, находящихся в критических состояниях, значительно увеличивает летальность.

В зарубежной медицинской литературе широко обсуждается проблема, получившая название «Abdominal compartment syndrome»-ACS, которую рассматривают, как одно из тяжелых проявлений распространенного перитонита (РП) [11, с. 21; 12]. Одной из основных причин развития компартмент-синдрома авторы считают ИАГ. 
В 2004 г. на конференции WSACS ИАГ была определена как: это стойкое повышение ВБД до 12 мм рт. ст. и более, которое определяется при трех стандартных измерениях с интервалом 4-6 часов [12].

В настоящее время определение СИАГ представляется так: это стойкое повышение ВБД >20 мм рт. ст. (с АПД <60 мм рт. ст.), которое ассоциируется с появлением новой органной недостаточности [12].

\section{Цель исслеАования}

Определение связи между уровнем интраабдоминальной гипертензии и распространенностью перитонита, а также определение оптимального способа декомпрессии.

\section{Материал и методы исслеАования}

Материалом настоящей работы являются результаты хирургического лечения 128 больных распространенным перитонитом (РП) в ГКБ им.С.С. Юдина в период с 2012-2018 годы. Средний возраст составил $48,4 \pm 8,8$ лет, при этом большую часть пациентов ( $n=93$ ) составляли лица трудоспособного возраста (до 50 лет), преимущественно мужчины (n=92). Дизайн исследования: ретроспективное, проспективное исследование.

В зависимости от способа завершения операции все больные с РП были разделены на 2 группы. В І-ю (контрольная) группу были включены 36 (28,1\%) пациентов, у которых лапаротомия заканчивалась глухим швом с традиционным дренированием; II-ю (основная) группу составили 92(71,9\%) больных с различными декомпрессивными вариантами ушивания лапаротомной раны. Первый вариант -ушивание в лапаротомной ране только кожи 29 (31,5\%) по Н.Н. Каншину; второй вариант-лапаростомия и декомпрессивное закрытие брюшной полости по D.H. Wittmann с постепенным сближением краев лапаротомной раны с помощью проленовой сетки у 17 больных; третий вариант -лапаростома на спицах у 32 (50,0\%) больных; четвертый вариант у 14 $(15,2 \%)$ больных- формирование Vас -ассистированой лапаростомы с применением терапии отрицательным давлением. Вначале использовали постоянный режим разряжения 90-125мм рт. ст. По мере очищения брюшной полости режим меняли на прерывистый 40-50-мм рт. ст. В среднем смена повязок производилась через каждые 2-3 дня.

Группы были сопоставимы по возрасту, полу и тяжести состояния. Сроки госпитализации, характер сопутствующих заболеваний, этиология и стадии перитонита сопоставимы в обеих исследуемых группах.
Больные относились к категории среднетяжелых, тяжелых и крайне тяжелых (средняя оценка тяжести со-

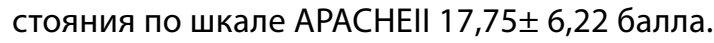

Критериями включения были:

- больные с распространенным перитонитом в токсической и терминальной стадии с тяжестью состояния по APACHEII выше 15 баллов и с органной дисфункцией по SOFA $>6$ баллов,

- больные с прогрессирующим и послеоперационным перитонитом,

- возраст больных от 18 до 78 лет.

В исследование не включали больных с панкреонекрозом, беременных и больных с тяжелыми сопутствующими заболеваниями в стадии декомпенсации.

Тяжесть состояния больных по АРACHEII <15 баллов принимали соответствующей реактивной стадии перитонита; тяжесть состояния до 23 баллов расценивали равной токсической стадии, а при оценке тяжести состояния >23 баллов, что обычно сопровождалось органной дисфункцией более трех органов, констатировали стадию декомпенсации (терминальльная стадия).

Средний балл по шкале SOFA к моменту поступления в стационар составил 6,8 балла.

В соответствии с целью программа исследований включала: определение клинико-лабораторных параметров, необходимых для ежедневной интегральной оценки, тяжести состояния по шкалам APACHEII и органной дисфункции SOFA; Оценка интраабдоминального давления в послеоперационном периоде ежедневно до 7 суток по завершении хирургического лечения; -определение связи между уровнем интраабдоминальной гипертензии и распространенностью воспалительного процесса брюшной полости; определение показателя абдоминально-перфузионого давления (АПД) -разница между средним артериальным и внутрибрюшным давлениями).

Методы исследования: Определение $\mathrm{PaO} 2, \mathrm{PaCO}$, $\mathrm{pH}$ артериальной крови (пункция бедренной артерии); концентрации электролитов плазмы при помощи аппарата «RapidLab 348»; определение гемоглобина и лейкоцитов при помощи гематологического счетчика «ABXMicros»; определение концентрации креатинина плазмы, общего билирубина на биохимическом анализаторе фирмы «Вауег»; определение лактата в сыворотке крови; контроль темпа мочеотделения.

ВБД мониторировали каждые 6 часов непрямым методом через уретральный катетер мочевого пузыря по М. Cheatham и соавт. (1998). 
Таблица 1. Характеристика взаимосвязи между величиной интраабдоминальной гипертензии, тяжестью физического состояния (APACHE II) и органной дисфункцией (SOFA) в сравниваемых группах перед операцией.

\begin{tabular}{|c|c|c|c|c|}
\hline \multirow{2}{*}{$\begin{array}{l}\text { Интегральные шкалы } \\
\text { Степень ИАГ }\end{array}$} & $\begin{array}{l}\text { 1-группа } \\
\text { (контрольная) }\end{array}$ & $\begin{array}{l}\text { 2-ягруппа } \\
\text { (основная) }\end{array}$ & $\begin{array}{l}\text { 1-ягруппа } \\
\text { (контрольная) }\end{array}$ & $\begin{array}{l}\text { 2-я группа } \\
\text { (основная) }\end{array}$ \\
\hline & \multicolumn{2}{|l|}{ APACHE II } & \multicolumn{2}{|l|}{ SOFA } \\
\hline $\begin{array}{l}\text { ВБД 12-15 мм рт.ст. } \\
\text { (ІСт.ИАГ) }\end{array}$ & \begin{tabular}{|l|}
$14,2 \pm 1,0$ \\
$3(8,3 \%)$ \\
\end{tabular} & \begin{tabular}{|l|}
$13,8 \pm 0,878$ \\
$7(7,6 \%)$ \\
\end{tabular} & \begin{tabular}{|l|}
$3,8 \pm 0,2$ \\
$3(8,3 \%)$ \\
\end{tabular} & $\begin{array}{l}3,6 \pm 0,4 \\
7(7,6 \%) \\
\end{array}$ \\
\hline $\begin{array}{l}\text { ВБД 16-20 мм рт.СТ. } \\
\text { (॥ст. ИАГ) }\end{array}$ & \begin{tabular}{|l|}
$18,4 \pm 1,8$ \\
$15(41,7 \%)$ \\
\end{tabular} & $\begin{array}{l}16,8 \pm 3,0 \\
31(33,7 \%) \\
\end{array}$ & \begin{tabular}{|l|}
$5,4 \pm 0,6$ \\
$15(41,7 \%)$ \\
\end{tabular} & \begin{tabular}{|l|}
$5,6 \pm 0,2$ \\
$31(33,7 \%)$ \\
\end{tabular} \\
\hline $\begin{array}{l}\text { ВБД21-25мм рт.Ст (IIIст. } \\
\text { ИАГ) }\end{array}$ & $\begin{array}{l}22,2 \pm 1,4 \\
11(30,6 \%) \\
\end{array}$ & $\begin{array}{l}21,8 \pm 3,0 \\
40(43,5 \%)\end{array}$ & $\begin{array}{l}5,4 \pm 0,6 \\
11(30,6 \%)\end{array}$ & \begin{tabular}{|l|}
$5,6 \pm 0,4$ \\
$40(43,5 \%)$ \\
\end{tabular} \\
\hline $\begin{array}{l}\text { ВБД>25мм рт.Ст. } \\
\text { (IVст. ИАГ) }\end{array}$ & $\begin{array}{l}28,4 \pm 1,2 \text { мм рт.ст. } \\
7(19,4 \%)\end{array}$ & $\begin{array}{l}\text { 26,6 } 2 \pm 2,8 \text { мм рт.ст. } \\
14(15,2 \%)\end{array}$ & $\begin{array}{l}\text { 8,4 } 42,6 \text { мм рт.ст. } \\
7(19,4 \%)\end{array}$ & $\begin{array}{l}8,8 \pm 2,2 \text { мм рт.Ст. } \\
14(15,2 \%)\end{array}$ \\
\hline
\end{tabular}

Статистическую обработку клинических наблюдений выполняли при помощи программы «EP linfo» на персональном компьютере Toshiba Satellite SA50492.

\section{Результаты \\ и обсу жАение}

До операции повышение ВБД было отмечено у всех 36 (100,0\%) больных первой группы. ИАГ І степени в I группе установлена у 9 (7,0\%) больных, II степени - у 10 (7,8\%), III степени - у 11 (8,6\%) и IV — 6 (4,7\%) больных. Синдром интраабдоминальной гипертензии (СИАГ) развился у 6 (4,7\%) пациентов.

Bo II группе ИАГ перед операцией была отмечена у 92 (100\%). Развитие СИАГ после операции на отмечали.

Повышение ВБД>15 мм рт. ст. приводило к ухудшению мезентериального кровообращения и характеризовалось снижением АПД ниже 85 мм рт. ст.

На Всемирном конгрессе по АКС (6-8 декабря 2004) предложили градацию ИАГ в следующем варианте (табл. 1).

В результате исследования величины ВБД у больных с распространенным перитонитом, осложненным перитонеальным сепсисом было установлено, что уровень ИАГ достоверно указывает на прогрессирование перитонита, увеличивается по мере клинического ухудшения состояния больных, что подтверждалось наличием клинических признаков перитонита, паралитической кишечной непроходимости и развившихся гнойно-септических осложнений, а также данными лабораторных тестов и оценкой тяжести состояния по интегральным шкалам APACHEII и SOFA (см. табл. 1).
Установлена статистически достоверная корреляционная связь между степенью ИАГ и ухудшением тяжести состояния по интегральным шкалам (АРACHE II) и SOFA ( $\mathrm{p}<0,05)$. Это было обусловлено прогрессированием перитонита, развитием перитонеального сепсиса и признаков полиорганной недостаточности.

При мониторинге ВБД у больных установлена положительная корреляция между ВБД и способом декомпрессии $(p<0,05)$.

Установлено, что при величине ВБД 16-20 мм рт. ст., наступает снижение АПД до 75-70 мм рт. ст., что приводит к ухудшению мезентериального кровообращения, а при величине ВБД болеет 25 мм рт. ст., происходит снижение АПД ниже 65 мм рт. ст., что приводит к выраженной гипоксии кишечника.

В дальнейшем установлено, что между уровнем ВБД и АПД в динамике имеется корреляционная зависимость: с увеличением ВБД уменьшается АПД, а при уменьшении ВБД, увеличивается АПД, что отражает нормализацию мезентериального кровообращения.

Таким образом, у больных с синдромом интраабдоминальной гипертензии, показатели внутрибрюшного и абдоминально-перфузионного давления являются прогностическими критериями, что необходимо учитывать при определении объёмов и вариантов инфузионной терапии и сроков выполнения оперативных вмешательств, направленных на декомпрессию кишечника и брюшной полости.

у 62 больных $(67,4 \%)$ основной группы необходимость реопераций была обусловлена невозможностью полного устранения источника перитонита в ходе первичной операции. Еще у 30 (32,6\%) больных эта необходимость была связана с картиной неклостридиального анаэробного перитонита. 

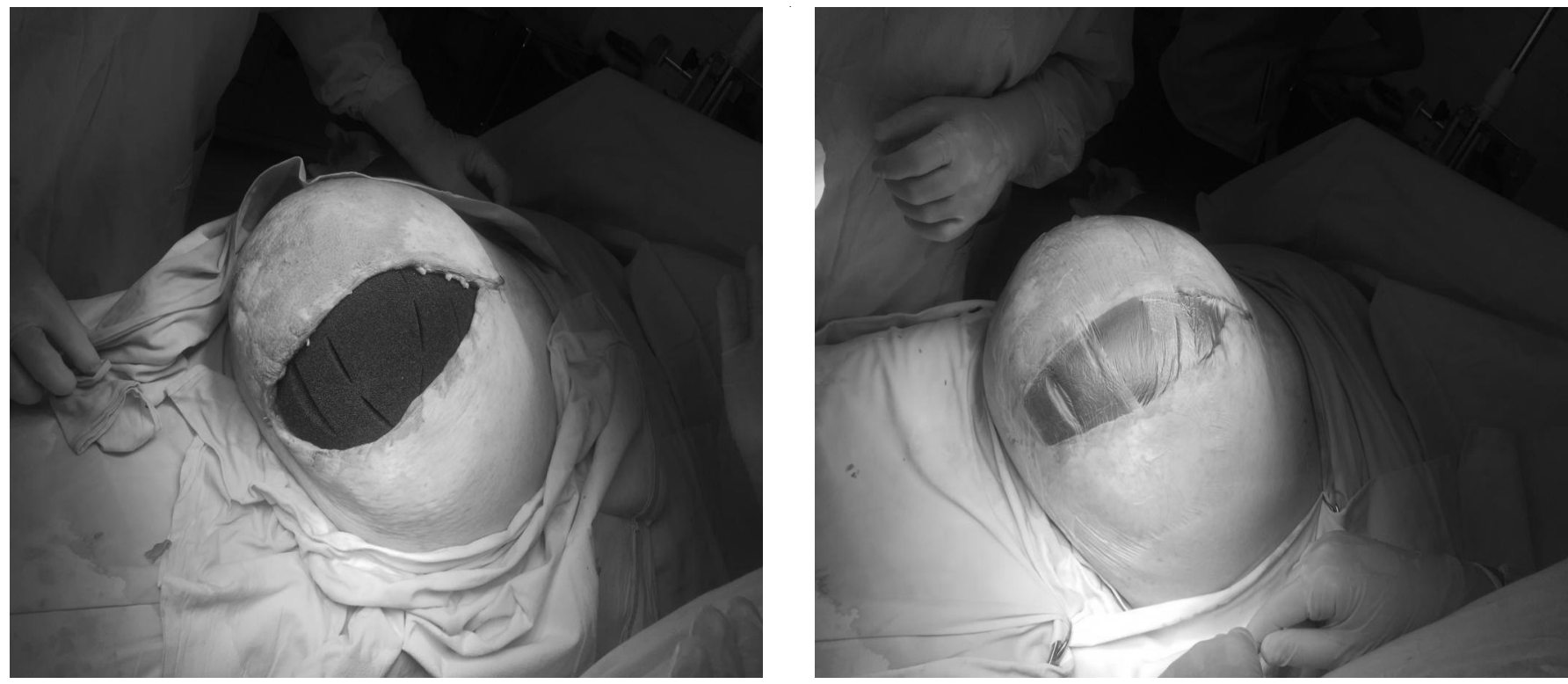

Рис. 1, Рис. 2. Этапы формирования Vас-системы предупреждало прогрессирование ИАГ и органную дисфункцию.

В целях профилактики ИАГ в послеоперационном периоде у 29 (31,5\%) больных использовали первый вариант декомпрессии брюшной полости - ушивание лапаротомной раны только кожными швами (Н.Н. Каншин, 1999; авт. свид. № 1050683). Полное отсутствие натяжения и благодаря этому снижение ВБД при ушивании лапаротомной раны только кожными швами дает при перитоните выраженный эффект. Этот вариант показан при программных реоперациях, субкомпенсированной динамической кишечной непроходимости (диаметр тонкой кишки до 5см), тяжести состояния по APACHEII более 16 баллов и степени органной дисфункции по SOFA 5-6 баллов у больных и ИАГ II-III степени и абдоминально перфузионном давлении 60 мм рт. ст. и менее.

Второй вариант - декомпрессивное закрытие лапаротомной раны по D.H. Wittmann применен у 17 (19,8\%) больных. Эта методика предусматривает постепенное сближение краев лапаротомной раны с помощью проленовой сетки. Временное закрытие лапаротомной раны при этом варианте увеличивает объем брюшной полости, за счет создаваемого диастаза мышечно-апоневротического слоя краев раны. Показаниями к этому варианту декомпрессии брюшной полости являются такие же как и при первом варианте декомпрессии: планируемые этапные санации, ИАГ ІІ-ІІІ степени, абдоминальное перфузионное давление менее 70 мм рт. ст. и менее, декомпенсированная степень динамической (паралитической) кишечной непроходимости - диаметр тонкой кишки 5см и более, тяжесть состояния по APACHEII более 19 баллов, 6 и более баллов по SOFA. Отличительной особенностью этого варианта являет- ся то, что при использовании второго варианта редко формируются вентральные грыжи. Вариант декомпрессии брюшной полости - лапаростомию на спицах мы применили у 46 (53,5\%) больных.

Показанием к открытой декомпрессии брюшной полости на спицах были такие же как при втором варианте, а также присутствие анаэробной неклостридиаьной инфекции.

При открытом ведении лапаротомной раны в течение последующей недели после операции наблюдается, что отек и дилатация петель кишок постепенно спадают, но одновременно с этим увеличивается латеральная мышечно-фасциальная ретракция. Для предупреждения этого недостатка необходимо перфорированную пленку закладывать между петлями кишок и париетальной брюшины влево и справа от срединной раны на 20-25 см.

Это позволит сохранить подвижность передней брюшной стенки за счет изоляции ее от сращений с конгломератом петель тонкой кишки и создаст возможность без значительного натяжения ушить лапаростому.

у 14 наиболее тяжелых больных (APACHEII >28 и SOFA > 12 баллов операция была завершена формированием лапаростомы с Vас-системой (рис. 1-4). По мере очищения брюшной полости режим разрежения меняли на прерывистый - 40-50-мм рт. ст. В среднем смена повязок производилась через каждые 2-3 дня. 

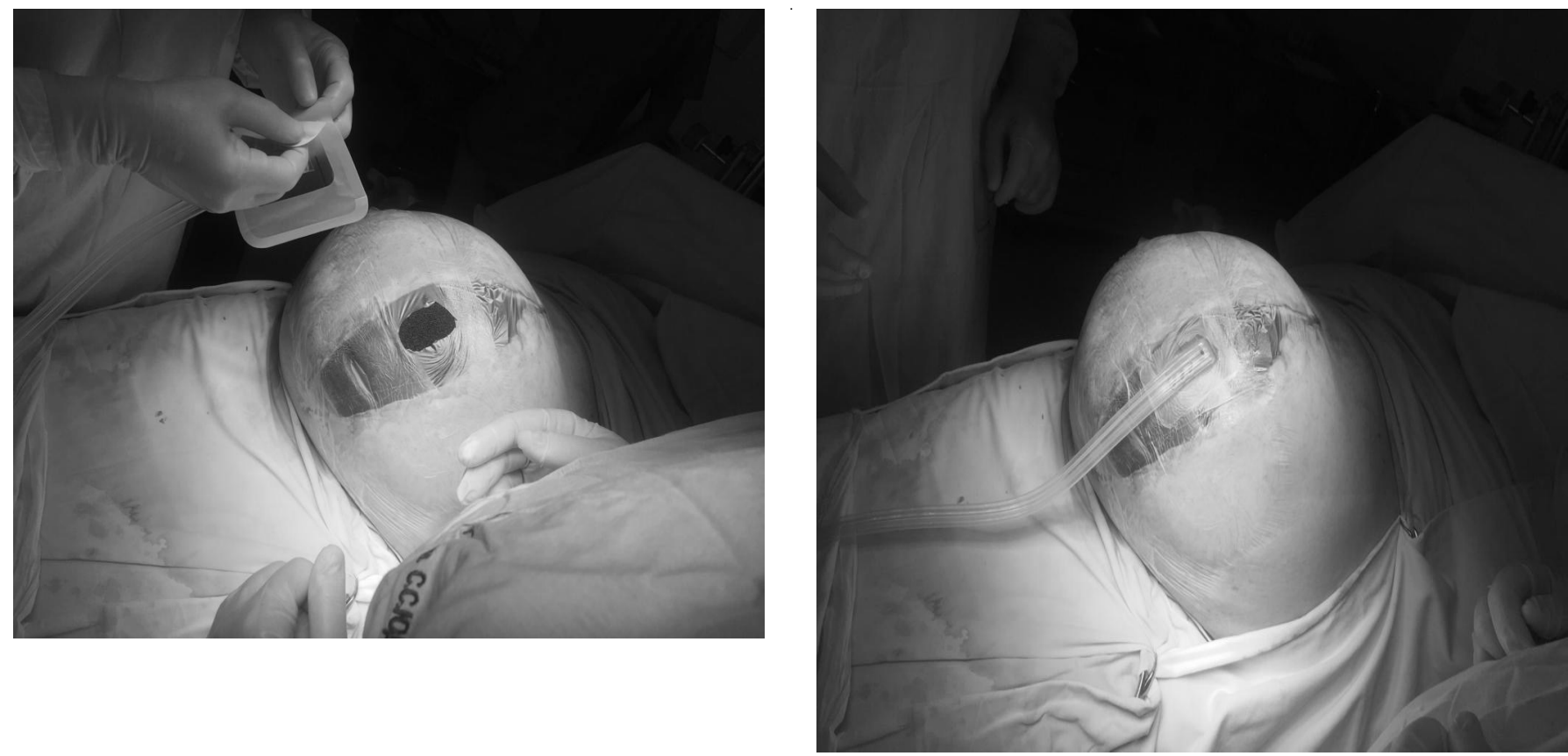

Рис. 3, Рис. 4. Окончание формирования Vас-системы

Уровень ВБД после использования методики D.H.Wittmann и лапаростомии на спицах свидетельствовал о более выраженном эффекте декомпрессии брюшной полости по сравнению с больными, которым выполняли декомпрессию ушиванием лапаротомной раны только кожи.

Использование II и III варианта декомпрессии по сравнению с I вариантом способствовало более

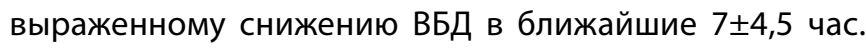
до $13,2 \pm 3,8$ мм рт. ст., что

Применение терапии отрицательным давлением (NPWT) у больных с распространенным перитонитом обеспечивает быстрое и контролируемое снижение внутрибрюшного давления, что является профилактикой развития абдоминального компартмент-синдрома и соответственно предотвращает развитие полиорганной дифункции.

Следует отметить, что степень органной дисфункции определяет и сроки закрытия лапаростомы. При наличии дыхательной, сердечной недостаточности и ВБД более 15 мм рт. ст., возникающих в результате сведения краев лапаротомной раны (после устранения перитонита), следует воздержаться от традиционного ушивания лапаротомной раны и использовать один из предложенных вариантов декомпрессивного закрытия брюшной полости.

При традиционном лечении в 1-й группе умерли 16(44,4\%); в группе с декомпрессивным ушиванием ла- паротомной раны умерли 20 (21,7\%) больных. Общая летальность среди 128 больных с распространенным перитонитом составила $(28,1 \%)$.

Основная причина смерти среди больных с декомпрессией брюшной полости - нозокомиальная пневмония - умерли 16 (17,4\%) пациентов; 4 (4,3\%) больных умерли в результате ухудшения сопутствующей сердечно-сосудистой недостаточности.

Главным и определяющим при выборе способа декомпрессивного завершения операции и программы этапных реопераций у больных с РП является результаты макроскопической оценки брюшины, морфо-функциональное состояние тонкой кишки, тяжесть состояния больного по АРACHE II (16 и более баллов), степень органной дисфункции по шкале SOFA(6 и более баллов) и уровень ИАГ (более 16 мм рт.ст.).

Таким образом, ушивание лапаротомной раны наглухо у больных с распространенным перитонитом явилось атрибутивно значимым фактором, который повышал риск развития ИАГ, интраабдоминальных осложнений и летального исхода.

Анализ результатов лечения тяжелых форм распространенного перитонита показал, что выбор в самом начале тактики пролонгированной декомпрессии с программными санациями как альтернатива традиционному хирургическому лечению достоверно демонстрируют лучшие показатели. 


\section{ЛИТЕРАТУРА}

1. Гельфанд Б.Р., Абдоминальный сепсис: современный взгляд на не стареющую проблему Вестник интенс. терапии 1997.

2. Гельфанд Б.Р., Проценко Д.Н., Игнатенко О.В., Ярошецкий А.И. Синдром интраабдоминальной гипертензии (Обзор литературы). // Consilium medicum 2005.7 (1)

3. Гельфанд Б.Р., Проценко Д.Н., Игнатенко О.В. и др. Синдром интраабдоминальной гипертензии у хирургических больных: состояние проблемы в 2007. // Инфекции в хирургии. Т. 5. 2007. № 3.

4. Гельфанд Б.Р. Доказательная медицина в клинической практике // «Инфекции в хирургии 2008 г. том 6. № 4.

5. Губайдуллин Р.Р. Нарушения в системе транспорта кислорода и пути их коррекции у больных с внутрибрюшной гипертензией. // Дис... д-ра мед. наук М., 2005.

6. Земляков Д.С. Коррекция внутрибрюшной гипертензии при неотложных и программных релапаротомиях. Диссер.к.м.н. 2016, Волгоград

7. Подачин П.В., Чубченко С.В. Показания к этапному хирургическому лечению распространенного перитонита. // Второй конгресс московских хирургов «Неотложная и специализированная хирургическая помощь» Тезисы докладов. 2007.

8. Савельев В.С., Гельфанд Б.Р., Филимонов М.И. и др. // Хирургическое лечение перитонита В кн.: Перитонит «Литтерра». 2006

9. Чадаев А.П., Хрипун А.И. М. Перитонит и внутрибрюшное давление М. 2003.

10. Diebel LN, Dulchavsky SA, Wilson RF. Effect of increased intra — abdominal pressure on mesenteric arterial and intestinal mucosal bloodflow. J. Trauma 1992;1.

11. Cortes-Puentes G., Gard K., Adams A. et al. Value and limitations of transpulmonary pressure calculations during intraabdominal hypertension. Crit. Care Med. 2013; 41 (8).

12. Cheatham ML, Ivatury RR, Malbrain ML, Sugrue M (2006) Options and challenges for the future. In: Ivatury R, Cheatham M, Malbrain M, Sugrue M (eds) Abdominal Compartment Syndrome. Landes.

13. Cheatham M.L., White M.W., Sagraves S.G., Johnson J.L., Block E.F. Abdominal perfusion pressure: a superior parameter in the assessment of intra-abdominal hypertension // J. Trauma. 2000 0ct. 49(4). 621-6; discussion.

14. Ivy M.E. et al. 2000 Intra-abdominal hypertension and abdominal compartment syndrome in burn patients // J Trauma. Vol. 49.

15. Khan S., Verma A., Ahmad S. et al. Analyzing intraabdominal pressures and outcomes in patients undergoing emergency laparotomy. J. Emerg. Trauma Shock. 2010; 3 (4).

16. Kitano Y., Takata M., Sasaki N. et al. Influence of increased abdominal pressure on steadystate cardiac performance // J Appl Physiol. 1999. -Vol. 86.

17. Malbrain M.L. Abdominal pressure in the critically ill. // Curr Opin Crit Care. 2000. 6.

18. Malbrain M.L., Jones F. Intra-abdominal pressure measurement technigues. In: Ivatury R., Cheabam M., Malbrain M., Sugrue M (eds) Abdominal Compartment Syndrome. Landes Bioscience, Georgetown. 2006.

19. Mayberry J.C., Goldman R.K., Mullins R.J., Brand D.M., Crass R.A., Trunkey D.D. (1999) Surveyed opinion of American trauma surgeons on the prevention of the abdominal compartment syndrome. J. Trauma 47.

20. Schein M., Wittman D.H., Aprahamian C.C., Condon R.E. The abdominal compartment syndrome: the physiological and clinical consequences of elevated intraabdominal pressure // J. Am. Col. Surg. 1995. Vol. 180.

21. Sukhotnik I., Mogilner J., Hayari L. et al. Effect of elevated in- tra-abdominal pressure and on superior mesenteric artery blood flow and enterocyte turnover in a rat. Pediat. Surg. Int. 2008; 24 (12).

22. Schneider C.G., Scholz J., Izbicki J.R. Abdominal compartment syndrome //Anasthesiollntensiv med Notfall med Schmerzther. 2000. Vol. 35.

23. Schein M., Wittman D.H., Aprahamian C.C., Condon R.E. The abdominal compartment syndrome: the physiological and clinical consequences of elevated intraabdominal pressure // J. Am. Col. Surg. 1995 Vol. 180.

24. Wittmann D.H., Schein M., Condon R.E. Management of secondary peritonitis [Review] // Annals of Surgery. 1996. Vol.224.

( ) Багдасарова Елена Анатольевна, Елеев Алим Анатольевич ( Alimeleev@gmail.com ), Багдасаров Валерий Вартанович.

Журнал «Современная наука: актуальные проблемы теории и практики» 